\title{
Pathology of iris in leprosy
}

\author{
Ebenezer Daniel, Gigi J Ebenezer, Charles K Job
}

\begin{abstract}
Aim-The histopathological features of the iris in leprosy were studied by light microscopy.

Method-Formalin fixed and paraffin embedded iris tissue excised during cataract surgery from 20 leprosy patients were sectioned and studied with haematoxylin and eosin stain and modified Fite Faraco's stain for acid fast bacilli (AFB).

Results-Chronic inflammatory reactions were seen in the iris of 11 patients, seven of whom did not have any clinically demonstrable evidence of iridocyclitis. Smooth muscle disruption and destruction were seen in two specimens. AFB were found in the iris tissue of a polar lepromatous patient whose skin smears were negative for AFB and who had completed the WHO recommended antileprosy multidrug therapy (MDT).

Conclusion-Histopathology discloses far more silent chronic iridocyclitis in leprosy patients than are diagnosed clinically. AFB can persist in the iris tissue even after completion of MDT. Smooth muscle disruption and destruction, a cause of the miotic pupil in leprosy has been conclusively demonstrated histopathologically. (Br F Ophthalmol 1997;81:490-492)
\end{abstract}

Branch of
Ophthalmology, Schieffelin Leprosy

Research and Training Centre, Karigiri, Tamilnadu, India

E Daniel

Department of Histopathology and Experimental

Pathology, Schieffelin

Leprosy Research and

Training Center,

Karigiri, North Arcot

District, Tamilnadu,

India - 632106

G J Ebenezer

C K Job

Correspondence to:

Dr Ebenezer Daniel, Branch of Ophthalmology,

Schieffelin Leprosy Research

and Training Center,

Karigiri, North Arcot

District, Tamilnadu, India -

632106 .

Accepted for publication 14 January 1997

Leprosy is a disease that affects many organs in the body. Mycobacterium leprae which causes the disease has a preference for certain body sites such as the skin, the peripheral nerves, and the nasal mucosa. The bacilli can also invade the anterior segment of the eye and many of the clinically observed ocular manifestations in leprosy have been attributed to the presence of the bacilli or its fragments in this organ. Very few clinicopathological studies exist in the field of ocular leprosy owing to the obvious fact that tissue from the eye, and especially within the eye, cannot be made easily available for histopathological studies. An instance where it is possible to get tissue from within the eye is during cataract extraction where the lens or part of the lens and a piece of iris tissue are removed. India which has the world's largest leprosy population ${ }^{1}$ also has the highest incidence of cataracts, ${ }^{2}$ and since the leprosy patient is as likely to get an age-related cataract as his or her non-leprosy counterpart a fairly large number of cataract extractions are done among leprosy patients. Despite this, in very few places have there been histopathological examinations performed on the tissues taken out during surgery. We report here the histopathological features observed in the iris tissue of leprosy patients who had undergone cataract extraction in our institution.

\section{Materials and methods}

On average, annually, 60 leprosy patients undergo cataract surgery at the Schieffelin Leprosy Research and Training Centre, Karigiri, south India. During surgery an iridectomy is done and the iris tissue obtained by this procedure is sent for histopathological examination. Twenty such consecutive iris specimens obtained from 20 patients underwent histopathological examination during January to March 1996. These iris specimens were fixed in $10 \%$ neutral formalin soon after removal and processed for paraffin section. Serial sections of $5 \mu \mathrm{m}$ thickness were made and some were stained with haematoxylin and eosin for routine histopathological examination; others with a modified Fite Faraco stain for acid fast bacilli. ${ }^{3}$ The sections stained with haematoxylin and eosin were examined for evidence of inflammation and other histological changes. The sections stained with modified Fite's stain were examined under an oil immersion lens for acid fast bacilli.

Charts of the patients from whom the tissues were taken were reviewed for demographic ocular complications. The patients were classified according to Ridley and Jopling's classification. ${ }^{4}$ Out of the 20 leprosy patients in whom iris histopathologically was studied there were six females and 14 males. The age of these patients ranged from 35 years to 71 years with a mean age of 60 years. The duration of the leprosy ranged from 9 years to 51 years with a mean of 23.5 years. Two of the patients belonged to the polar tuberculoid group (TT), three to the borderline tuberculoid (BT), five to the borderline lepromatous (BL), and the remaining 10 to the polar lepromatous group (LL). The iris tissue was taken from 13 right eyes and seven left eyes.

In 15 patients a peripheral or a button hole iridectomy was done during the cataract extraction. In the other five patients a complete 


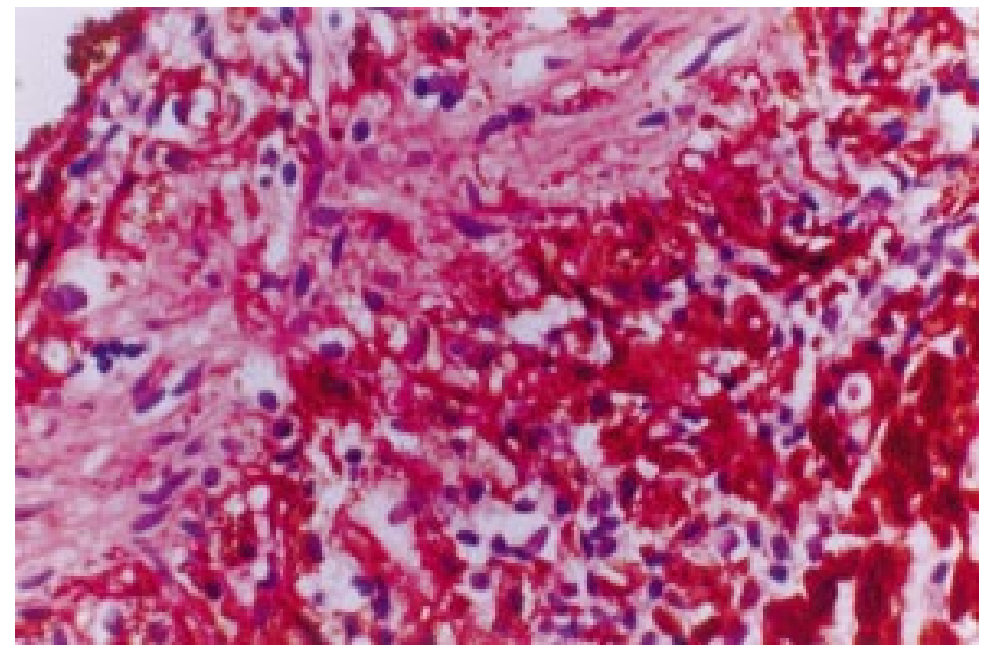

Figure 1 Photomicrograph of iris stromal tissue infiltrated with lymphocytes, plasma cells, and pigmented macrophages. (Haematoxylin and eosin, magnification $\times 560$.)

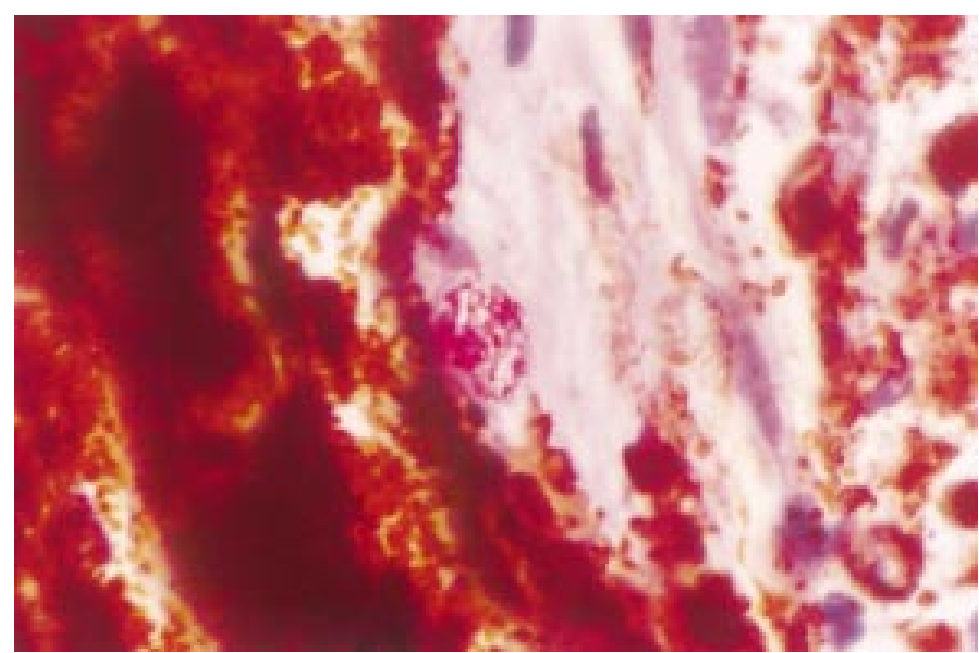

Figure 2 Photomicrograph showing a clump of acid fast bacilli within a macrophage in the iris stroma. (Modified Fite Faraco stain $\times$ 700.)

or a broad based iridectomy had to be done as a result of ocular complications such as posterior synechiae, small undilating pupil, and iris atrophy.

Of the five tuberculoid patients (TT and BT), two had completed the multidrug therapy (MDT) as recommended by the $\mathrm{WHO}^{5}$ and the remaining three had had dapsone monotherapy only. Of the 15 lepromatous patients (BL and LL) eight had completed MDT, four were on treatment with MDT, and three had had only dapsone monotherapy. Although eight of the lepromatous patients had been smear positive during the course of their disease, only three of them had acid fast bacilli positive skin smears at the time of surgery. Two borderline lepromatous patients gave history of having had a reversal or a type I reaction and one LL patient had had an erythema nodosum leprosum or a type II reaction. No other history of any reactive episodes could be elicited from the patients' charts. Features of old iridocyclitis such as iris atrophy, old keratic precipitates, and posterior synechiae were seen in five lepromatous patients.

\section{Results}

Chronic inflammation characterised by dense infiltration of the iris stromal tissue with collections of lymphocytes, histiocytes, plasma cells, and pigmented macrophages (Fig 1) was seen in nine $(45 \%)$ out of the 20 iris specimens. Of these eight were from lepromatous patients and one was from a BT patient. Four of the patients $(44 \%)$ had features of old iridocyclitis that could be proved clinically by slit-lamp biomicroscopic examination.

One other patient gave a history relating to past episodes of iridocyclitis. Two of the polar lepromatous patients were skin smear positive for acid fast bacilli at the time of iris biopsy. All the others were skin smear negative for acid fast bacilli at the time of biopsy. In one specimen only were acid fast bacilli seen in the iris tissue. Two of the patients who had chronic inflammation also had smooth muscle disruption and destruction.

Smooth muscle destruction, demonstrated by the disruption and destruction of smooth muscle fibres by the infiltration of inflammatory cells (Fig l) was seen in two of the biopsy specimens. Both were LL patients whose skin smears were negative for acid fast bacilli. Both exhibited clinical features of old iridocyclitis. One of these patients also had acid fast bacilli in the iris.

In one specimen acid fast stain showed a clump of acid fast bacilli within a macrophage adjacent to the pigmented epithelium (Fig 2). The presence of definite acid fast bacilli was demonstrated in only one patient who was skin smear negative for acid fast bacilli. This patient also had smooth muscle destruction as well as clinical signs of old iridocyclitis. In one other patient acid fast fragments were seen but no definite bacilli could be made out.

Evidence of iris atrophy was seen in four specimens which had blood vessels with thick hyalinised walls. None of these patients exhibited the iris atrophy clinically.

\section{Discussion}

The involvement of iris tissue in leprosy is well known. Despite this very few histopathological studies have been done on the iris tissue of leprosy patients.

Our finding that there is chronic inflammatory pathology in the iris tissue of nearly $56 \%$ of patients who do not give a history of iridocyclitis or show features of old iridocyclitis suggests that silent chronic iridocyclitis is a far more extensive feature of ocular leprosy than is thought. ${ }^{6}$ Except for one patient, all who exhibited this feature were lepromatous patients (BL and LL). The exception was a borderline tuberculoid patient. This was unusual as tuberculoid patients are not usually known to have inflammatory reactions within the eye. One plausible explanation was that this patient had been wrongly classified or downgraded towards the lepromatous side. Reassessment of the patient and his records negated this explanation. The other possibility is that the patient may have had other pathologies such as other granulomatous infections or collagen related diseases that could have elicited the 
inflammatory reaction in the iris tissue. These were not investigated for in this patient. Another interesting aspect of the chronic inflammation found within the iris of this borderline tuberculoid patient was that no epithelioid granulomas that are a characteristic feature of tuberculoid leprosy were found.

The entry of Mycobacterium leprae into the anterior segment of the eye is presumed to be through the blood vessels of the ciliary body. Although the presence of acid fast bacilli has been demonstrated in the eyes of leprosy patients in previous studies, ${ }^{78}$ information is not available as to what happens to these bacilli after antileprosy treatment. The patient in whom the iris tissue exhibited one clump of acid fast bacilli had been treated with adequate antileprosy drugs as recommended by the WHO and had been released from treatment several years ago. He was negative for acid fast bacilli in the routine skin smear sites. $M$ leprae persisting in fibrosed nerves, long after patients had been declared disease arrested following antileprosy therapy, has been reported. ${ }^{9}$ These persisting organisms as a nidus for recrudescence of the disease is also well known. It is possible that in most patients the organisms invading the iris tissue disappear during the course of treatment and on rare occasions such as this patient the bacilli may persist inside macrophages and also possibly inside smooth muscle cells. These persisting organisms and their antigens could be responsible for recurrent reactions occurring in the eyes after they have become negative for acid fast bacilli in the skin and even be responsible for an occasional case of relapse. ${ }^{10}$ To our knowledge this is the first report of acid fast bacilli in the iris tissue of a leprosy patient who had completed the fixed duration multidrug therapy as recommended by the WHO. It would be interesting to see whether more acid fast bacilli can be demonstrated in slide preparations that have had the intense iris pigment that could obscure acid fast bacilli removed.

Clinically, atrophy of the iris and the small pupil are well established features of ocular leprosy. In many lepromatous patients with long standing disease this is a common feature. The smooth muscle destruction by infiltration of inflammatory cells was demonstrated in two patients who belonged to the lepromatous group and who had evidence of past iridocyclitis. The iris tissue excised from near the root of the iris provides proof of the destruction of the dilator pupillae that would lead to a miotic pupil.

Patients who belong to the polar and borderline lepromatous groups are prone to ocular complications that are related to the presence of the organism or its fragments in the eye. Additional ocular therapy may help prevent such complications in these patients. Ofloxacin is a fluorinated 4-quinolone antibiotic that is effective against a wide spectrum of Gram positive and Gram negative organisms and has been shown to penetrate into the aqueous in concentrations that exceed the minimum inhibitory concentration for many of these bacteria. ${ }^{11}$ This drug has been found to be effective against $M$ leprae ${ }^{12}$ but its efficacy in penetrating into the aqueous of leprosy patients is not known. It is also available as topical eyedrops and may be a useful adjunct in ocular leprosy therapy.

We are grateful to Mr R Soundarajan, histopathology technician, for his excellent technical assistance.

1 Dharmshaktu NS. Prospects of elimination of leprosy in India. Ind F Lepr 1994;66:11-8.

2 Minassian DC, Mehra V. 3.8 Million blinded by cataract each year: projections from the first epidemiological study each year: projections from the first epidemiological study
of incidence of cataract blindness in India. Br f Ophthalmol of incidence of cat $1990 ; 74: 341-3$.

3 Job CK, Chacko CJG, A modified Fite's stain for demonstration of $\mathrm{M}$ leprae in tissue sections. Ind $\mathcal{F}$ Lepr 1986;58:17-8

4 Ridley DS, Jopling W H. Classification of leprosy according to imunity; a five group system. Int $\mathcal{F}$ Lepr 1966;34:255-73.

5 World Health Organisation Study Group. Chemotherapy of leprosy for control programmes. World Health Organisation. Technical Report Series No 675. Geneva: WHO, 1982

6 Espiritu CG, Gelber R, Ostler HB. Chronic anterior uveitis in leprosy: insidious cause of blindness. $\mathrm{Br} \mathcal{F}$ Ophthalmol 1991;75:273-5.

7 Desikan KV, Job CK. Report of study of eye lesions in three autopsied cases of leprosy. Leprosy in India 1970;42:1-3.

8 Hashizume H, Shionuma E. Electron microscopic study of Hashizume H, Shionuma E. Electron microscopic study of

9 Job CK, Victor DBI, Chacko CJG. Progressive nerve lesion in a disease arrested leprosy patient-an electron microscopic study. Int F Lepr 1977;45:255-60.

10 Joffrion Van C. Ocular leprosy. In: Hastings RC, ed. Leprosy. 2nd ed. New York: Churchill Livingstone, 1994:357.

11 Guten SV, Lew D, Paccolat F, Vandaux P, Brazititos PD, Leuenbergen PM. Aqueous humor penetration of ofloxacin given by various routes. Am $\mathcal{F}$ Ophthalmol 1994;177:87-9.

2 Franzblau SG, White KE. Comparative in vitro activities of 20 fluoroquinolones against Mycobacterium leprae. Antimicrob Agents Chemother 1990;34:229-31. 